\title{
EMISSÕES OTOACÚSTICAS EVOCADAS TRANSIENTES EM RECÉM-NASCIDOS A TERMO E PRÉ-TERMO
}

\author{
Transient evoked otoacoustic emissions \\ in full-term and preterm newborns
}

\author{
Ana Dolores Passarelli de Melo(1), Kátia de Freitas Alvarenga (2), Daniela Jovel Modolo ${ }^{(3)}$, \\ Maria Cecília Bevilacqua ${ }^{(4)}$, Andréa Cintra Lopes ${ }^{(5)}$, Raquel Sampaio Agostinho-Pesse ${ }^{(6)}$
}

\begin{abstract}
RESUMO
Objetivo: analisar a amplitude absoluta e nível de Response das emissões otoacústicas evocadas transientes em recém-nascidos pré-termo e a termo. Métodos: participaram deste estudo 50 recémnascidos a termo e 50 pré-termo, os quais foram submetidos à triagem auditiva neonatal por meio da pesquisa das emissões otoacústicas evocadas transientes, realizada entre 24 horas e 11 semanas de vida. Todos os recém-nascidos apresentaram presença de emissões otoacústicas evocadas por clique no teste da triagem auditiva neonatal. Resultados: não houve diferença estatisticamente significante da amplitude e nível de Response entre os recém-nascidos a termo e pré-termos. Não houve correlação significante entre os fatores prematuridade, idade do recém-nascido no teste e tempo de estimulação auditiva com os resultados obtidos. Conclusão: as emissões otoacústicas evocadas transientes registradas em recém-nascidos a termos e pré-termos apresentam semelhante amplitude absoluta e nível de Response.
\end{abstract}

DESCRITORES: Audiologia; Triagem Neonatal; Prematuro

\section{INTRODUÇÃO}

A pesquisa das emissões otoacústicas evocadas transientes (EOE-t) é um procedimento

(1) Fonoaudióloga; Faculdade de Odontologia de Bauru da Universidade de São Paulo, FOB-USP, Bauru, SP, Brasil; Mestre em Fonoaudiologia pela Faculdade de Odontologia de Bauru da Universidade de São Paulo.

(2) Fonoaudióloga; Professora Livre Docente do Departamento de Fonoaudiologia pela Faculdade de Odontologia de Bauru da Universidade de São Paulo, FOB-USP, Bauru, SP, Brasil.

(3) Fonoaudióloga; Faculdade de Odontologia de Bauru da Universidade de São Paulo, FOB-USP, Bauru, SP, Brasil; Mestre em Fonoaudiologia pela Faculdade de Odontologia de Bauru da Universidade de São Paulo.

(4) Fonoaudióloga; Professora Titular do Departamento de Fonoaudiologia da Faculdade de Odontologia de Bauru da Universidade de São Paulo, FOB-USP, Bauru, SP, Brasil.

(5) Fonoaudióloga; Professora Doutora do Departamento de Fonoaudiologia pela Faculdade de Odontologia de Bauru da Universidade de São Paulo, FOB-USP, Bauru, SP, Brasil.

(6) Fonoaudióloga; Faculdade de Odontologia de Bauru da Universidade de São Paulo, FOB-USP, Bauru, SP, Brasil; Especialização em Audiologia pelo Instituto de Comunicação e Audição de Bauru.

Conflito de interesses: inexistente objetivo, não invasivo e rápido, comumente utilizado em programas de Triagem Auditiva Neonatal (TAN) ${ }^{1}$. Para o registro confiável das EOE-t são necessárias condições favoráveis da orelha externa e média ${ }^{1,2}$ assim como, um ambiente tratado acusticamente ou silencioso.

Neste contexto, questiona-se também, se a prematuridade deveria ser uma variável a ser considerada na análise do resultado obtido na TAN com EOE-t, uma vez que sua realização é limitada em recém-nascidos (RN) com menos de 35 semanas de gestação, principalmente entre 28 e 30 semanas, devido a dimensão do meato acústico externo e eventual imaturidade do desenvolvimento da cóclea ${ }^{3}$.

Estudos demonstraram que RN prematuros apresentam presença de EOE-t a partir da $27^{\mathrm{a}}$ semana de gestação. Contudo, constataram que os $\mathrm{RN}$ a termo apresentaram maior índice de resultado passa na triagem auditiva neonatal, e ressaltaram que a pressão da orelha média, obstrução do meato acústico externo, e o próprio desenvolvimento da orelha externa e média podem ter influenciado nos resultados ${ }^{3,4}$. Os autores ressaltaram que os 
RN pré-termo geralmente necessitam de cuidados especiais como unidade de terapia intensiva na qual recebem intervenção por meio de incubadora, medicação ototóxica e ventilação mecânica. Desta maneira, os autores ressaltaram que a prematuridade constitui em potencial um indicador de risco para alterações auditivas ${ }^{4}$.

$\mathrm{Na}$ literatura da área tem sido reportada a assimetria existente no sistema auditivo principalmente entre a orelha direita e esquerda, por meio da pesquisa das emissões otoacústicas. Os estudos realizados com crianças ${ }^{5}$, e com recém-nascidos, tanto termo quanto pré-termo ${ }^{6-9}$, demonstraram maiores valores de amplitude média das EOE-t na orelha direita quando comparados à esquerda ${ }^{10}$. Em contrapartida, outros estudos não encontraram diferença significante entre as oreIhas direita e esquerda, na pesquisa das EOE-t em recém-nascidos a termo e pré-termo ${ }^{11-14}$, com semelhante achado na pesquisa das emissões otoacústicas por produto de distorção (EOE-PD) ${ }^{15}$. A assimetria entre as orelhas tem sido relacionada às diferenças anatômicas do sistema auditivo, à lateralidade e gênero, assim como, pela atuação eferente do sistema auditivo distinta para as orelhas ${ }^{10}$.

A amplitude das EOE-t dos recém-nascidos nas bandas de altas frequências está associada à idade pós-concepcional no momento do teste (entre $31 \mathrm{e}$ 36 semanas de gestação para o grupo pré-termo, e de 37 a 44 semanas no grupo termo), ou seja, quanto maior a idade concepcional maior a amplitude das emissões otoacústicas ${ }^{6,10}$. Os autores ressaltaram que o parâmetro amplitude nas EOE-t fornece evidências da presença das emissões otoacústicas e também pode ser considerado como indicativo de maturação do sistema auditivo periférico em recém-nascidos ${ }^{6,10}$.

Estudos longitudinais descreveram a associação entre a idade pós-concepcional e a amplitude das EOE-t ${ }^{16,17}$, quando considerado o tempo de vida em horas, entre 3 e 238 horas ${ }^{18}$; ou em dias, a partir do $2^{\circ}{ }^{19}$ ou $4^{\circ}$ dia de vida ${ }^{20,21}$. Por outro lado, esta associação não foi observada quando se considerou 0 tempo de vida entre 24 e 54 horas ${ }^{22,23}$. Entretanto, apenas um estudo demonstrou diferença estatisticamente significante entre a amplitude das EOE-t em recém-nascidos a termo e pré-termo ${ }^{17}$.

Assim, o objetivo deste trabalho foi analisar a amplitude absoluta por banda de frequência e nível de Response das emissões otoacústicas evocadas transientes em recém-nascidos pré-termo e a termo, com idade pós-concepcional entre 24 horas de vida a 11 semanas.

\section{MÉTODOS}

No programa de Triagem Auditiva Neonatal (TAN) do Hospital Maternidade Santa Isabel da cidade de Bauru - SP, no qual este estudo foi realizado, a triagem auditiva é realizada em duas etapas: teste, antes da alta hospitalar e o reteste para os recém-nascidos que não-passaram, no máximo até 20 dias após o teste. O programa de TAN é de responsabilidade do Departamento de Fonoaudiologia, da Faculdade de Odontologia de Bauru / Universidade de São Paulo, no qual está inserido o Projeto Modelo de Saúde Auditiva no Recém-Nascido (FAPESP - 2003/06415-6).

Foram selecionados para participar deste estudo, os recém-nascidos a termo e pré-termo, nascidos no período de janeiro a setembro de 2005, que apresentaram função normal de células ciliadas externas, confirmada pela presença de EOE-t, com promediação de 2080 cliques. Neste período nasceu um total de 1403 recém-nascidos e foram avaliados 1312, correspondendo a $93,51 \%$ dos nascimentos da maternidade. Os $91(6,48 \%) \mathrm{RN}$ restantes não concluíram ou não realizaram a TAN.

Participaram deste estudo 50 recém-nascidos a termo, sendo 28 do sexo feminino e 22 do sexo masculino, e 50 pré-termo, sendo 22 do sexo feminino e 28 do sexo masculino. O grupo pré-termo foi subdividido em grupo A composto de 15 recémnascidos que apresentavam apenas o fator prematuridade, e o Grupo B por 35 recém-nascidos que apresentavam prematuridade associada a indicadores de risco para a deficiência auditiva de acordo com os critérios propostos por Joint Committe Infant Hearing (2000) ${ }^{24}$.

A TAN foi realizada nos recém-nascidos a termo antes da alta hospitalar e o reteste no dia do "Teste do Pezinho", na própria maternidade em torno de sete a 10 dias após a alta hospitalar. Já nos recémnascidos pré-termo a TAN foi realizada durante o período de internação no hospital, na alta hospitalar ou por meio de agendamento. No momento da triagem auditiva os recém-nascidos estavam dormindo ou em estado de sonolência após a amamentação, em ambiente silencioso, com controle do ruído ambiental. Não foi realizada a TAN em recém-nascidos com menos de 24 horas de vida ${ }^{25}$.

O equipamento utilizado foi o Madsen Capella, por meio da apresentação de clique não linear, na intensidade de 80 dB NPS. O critério de passa/nãopassa foi à reprodutibilidade da resposta coclear de no mínimo $70 \%$ de correlação e relação sinal/ruído de $6 \mathrm{~dB}$ NPS em 3 frequências, incluindo $4 \mathrm{kHz}$.

A resposta das EOE-t foi analisada por meio da amplitude absoluta e do valor de Response, sendo que este último representa o nível total de energia 
que se correlaciona nos traçados $A$ e $B$ obtidos do Fast Fourier Transforms (FFT). O FFT reflete o espectro da resposta que é comum aos traçados $A$ e $B$, sendo considerada a verdadeira emissões otoacústicas, sem contaminação do ruído de fundo ${ }^{1}$.

O estudo foi aprovado pelo Comitê de Ética em Pesquisa em Seres Humanos da Faculdade de Odontologia de Bauru da Universidade de São Paulo, processo no 114/2005.

Os resultados foram analisados por meio da análise estatística utilizando-se o Teste "t" Student a fim de comparar a média da amplitude das EOE-t dos recém-nascidos a termo e pré-termo em função das orelhas e do gênero, além da comparação entre Grupo A e B em função das orelhas. O teste estatístico coeficiente de correlação de Pearson, foi utilizado para a análise da correlação entre a prematuridade (semanas de gestação ao nascimento), idade do recém-nascido no teste (idade corrigida em semanas) e o tempo de estimulação auditiva (em semanas). Para ambos os testes foram adotados o nível de significância $p \leq 0,05$.

\section{RESULTADOS}

A Tabela 1 mostra a análise estatística do nível de Response obtido na pesquisa das EOE-t, considerando as orelhas, para os recém-nascidos pré-termo, comparando o Grupo A e o Grupo B. Como não foi observada diferença estatisticamente significante entre o nível de Response obtido nos recém-nascidos pertencentes ao grupo $A$ (apenas prematuridade) quando comparado ao grupo $B$ (prematuridade associada a outros indicadores de risco para a deficiência auditiva), a análise a seguir foi realizada considerando apenas os grupos de recém-nascidos a termo e pré-termo.

Nas Tabelas 2 e 3 encontra-se a análise comparativa das médias da amplitude absoluta por banda de frequência e do nível de Response, considerando as orelhas dos grupos de recém-nascidos a termo e pré-termo. Foi encontrada diferença significante entre as orelhas direita e esquerda para os grupos estudados. Assim, a análise realizada a seguir considerou as orelhas separadamente.

A Tabela 4 apresenta a análise comparativa por meio do Teste "t" Student, das EOE-t obtidas nos recém-nascidos a termo e pré-termo, considerando as médias da amplitude absoluta por banda de frequência e do nível de Response, de acordo com as orelhas.

A análise da correlação entre a amplitude absoluta por banda de frequência e dos níveis de Response das EOE-t e os fatores prematuridade, idade do recém-nascido no teste e tempo de estimulação auditiva, de acordo com as orelhas está apresentada nas Tabelas 5 e 6 (coeficiente de Correlação de Pearson). Não foram observadas correlações estatisticamente significantes entre a amplitude absoluta por banda de frequência e dos níveis de Response das EOE-t e as variáveis estudadas, para os recém-nascidos submetidos a triagem auditiva neonatal.

Tabela 1 - Análise das médias e desvios padrão do nível de Response das EOE-t nos recém-nascidos pré-termo do Grupo A e Grupo B em função das orelhas

\begin{tabular}{cccccc}
\hline & \multicolumn{2}{c}{ Grupo A } & \multicolumn{2}{c}{ Grupo B } & \multirow{2}{*}{$\mathbf{p}$} \\
\cline { 2 - 5 } & Média dB NPS & DP & Média dB NPS & DP & \\
\hline OD & 24,19 & 4,21 & 24,12 & 4,95 & 0,9634 \\
OE & 21,81 & 2,88 & 23,44 & 4,82 & 0,2289 \\
\hline
\end{tabular}

${ }^{*} p \leq 0,05$ : estatisticamente significante

Tabela 2 - Análise comparativa da amplitude absoluta por banda de frequência e nível de Response das emissões otoacústicas evocadas transientes obtidas nas orelhas direita e esquerda, para os recém-nascidos a termo

\begin{tabular}{ccccccc}
\hline & \multicolumn{7}{c}{ Banda de Frequência } & \multirow{2}{*}{ Response } \\
\cline { 2 - 6 } & $\mathbf{1 0 0 0}$ & $\mathbf{1 5 0 0}$ & $\mathbf{2 0 0 0}$ & $\mathbf{3 0 0 0}$ & $\mathbf{4 0 0 0}$ & \\
\hline OD & 12,12 & 14,39 & 15,94 & 18,69 & 18,50 & 25,15 \\
OE & 11,06 & 12,81 & 14,27 & 14,47 & 16,44 & 23,72 \\
\hline $\boldsymbol{p}$ & 0,22 & $0,04^{*}$ & $0,02^{*}$ & $0,04^{*}$ & $0,00^{*}$ & $0,00^{*}$ \\
\hline
\end{tabular}

${ }^{*} p \leq 0,05$ : estatisticamente significante 
Tabela 3 - Análise comparativa da amplitude absoluta por banda de frequência e nível de Response das emissões otoacústicas evocadas transientes obtidas nas orelhas direita e esquerda, para os recém-nascidos pré-termo

\begin{tabular}{ccccccc}
\hline & \multicolumn{7}{c}{ Banda de Frequência } & \multirow{2}{*}{ Response } \\
\cline { 2 - 6 } & $\mathbf{1 0 0 0}$ & $\mathbf{1 5 0 0}$ & $\mathbf{2 0 0 0}$ & $\mathbf{3 0 0 0}$ & $\mathbf{4 0 0 0}$ & \\
\hline OD & 10,95 & 13,94 & 15,13 & 17,98 & 17,51 & 24,14 \\
OE & 10,21 & 11,40 & 13,30 & 16,63 & 16,35 & 22,95 \\
\hline $\boldsymbol{p}$ & 0,39 & $0,00^{*}$ & $0,02^{*}$ & 0,06 & $0,05^{*}$ & $0,02^{*}$ \\
\hline
\end{tabular}

${ }^{\star} p \leq 0,05$ : estatisticamente significante

Tabela 4 - Análise comparativa da média da amplitude absoluta por banda de frequência e nível de Response das emissões otoacústicas evocadas transientes registradas nos recém nascidos a termo e pré-termo, de acordo com as orelhas

\begin{tabular}{ccccccc}
\hline \multicolumn{7}{c}{ Emissões Otoacústicas Evocadas Transientes ( $\mathbf{p})$} \\
\hline Termo e Pré-termo & $\mathbf{1 0 0 0}$ & $\mathbf{1 5 0 0}$ & $\mathbf{2 0 0 0}$ & $\mathbf{3 0 0 0}$ & $\mathbf{4 0 0 0}$ & Response \\
\hline Orelha direita & 0,28 & 0,69 & 0,47 & 0,49 & 0,40 & 0,29 \\
Orelha esquerda & 0,42 & 0,24 & 0,36 & 0,40 & 0,93 & 0,38 \\
\hline
\end{tabular}

${ }^{*} p \leq 0,05$ : estatisticamente significante

Tabela 5 - Correlação da amplitude absoluta por banda de frequência e nível de Response das emissões otoacústicas evocadas transientes em função da prematuridade, idade do recém-nascido no teste e tempo de estimulação auditiva para orelha direita, considerando os recém-nascidos submetidos a triagem auditiva neonatal

\begin{tabular}{ccccccc}
\hline & \multicolumn{7}{c}{ Orelha Direita } \\
\hline $\mathbf{r}$ & $\mathbf{1 ~ k H z}$ & $\mathbf{1 . 5} \mathbf{~ k H z}$ & $\mathbf{2 ~ k H z}$ & $\mathbf{3 ~ k H z}$ & $\mathbf{4 ~ k H z}$ & Response \\
$\mathbf{p}$ & $-0,019$ & 0,048 & 0,159 & 0,340 & 0,095 & 0,123 \\
$\mathbf{r}$ & 0,891 & 0,737 & 0,269 & $0,016^{*}$ & 0,508 & 0,393 \\
$\mathbf{p}$ & 0,155 & 0,023 & $-0,109$ & $-0,165$ & $-0,144$ & $-0,097$ \\
$\mathbf{r}$ & 0,281 & 0,869 & 0,447 & 0,250 & 0,317 & 0,499 \\
$\mathbf{p}$ & 0,178 & 0,028 & $-0,192$ & $-0,234$ & $-0,062$ & $-0,015$ \\
\hline
\end{tabular}

${ }^{*} p \leq 0,05$ : estatisticamente significante

Tabela 6 - Correlação da amplitude absoluta por banda de frequência e nível de Response das emissões otoacústicas evocadas transientes em função da prematuridade, idade do recém-nascido no teste e tempo de estimulação auditiva para orelha esquerda, considerando os recém-nascidos submetidos a triagem auditiva neonatal

\begin{tabular}{ccccccc}
\hline \multicolumn{7}{c}{ Orelha Esquerda } \\
\hline $\mathbf{r}$ & $\mathbf{1 ~ k H z}$ & $\mathbf{1 . 5} \mathbf{~ k H z}$ & $\mathbf{2 ~ k H z}$ & $\mathbf{3 ~ k H z}$ & $\mathbf{4 ~ k H z}$ & Response \\
$\mathbf{p}$ & 0,124 & 0,084 & 0,153 & 0,090 & 0,032 & 0,077 \\
$\mathbf{r}$ & 0,388 & 0,558 & 0,286 & 0,533 & 0,824 & 0,594 \\
$\mathbf{p}$ & $-0,005$ & 0,060 & $-0,024$ & $-0,097$ & 0,091 & $-0,015$ \\
$\mathbf{r}$ & 0,973 & 0,675 & 0,864 & 0,500 & 0,527 & 0,915 \\
$\mathbf{p}$ & 0,083 & $-0,136$ & $-0,111$ & 0,113 & 0,004 & 0,039 \\
\hline
\end{tabular}

${ }^{*} p \leq 0,05$ : estatisticamente significante 


\section{DISCUSSÃO}

A prematuridade constitui-se em potencial um indicador de risco para alterações auditivas, não pelo quadro em si, mas sim pelo fato do recém-nascido pré-termo geralmente necessitar de cuidados especiais nas unidades de terapia intensiva, como por exemplo, a intervenção por meio de incubadora, utilização de possíveis medicamentos ototóxicos e ventilação mecânica ${ }^{4}$.

Neste estudo, ao comparar o nível de Response das EOE-t nos recém-nascidos pré-termos que tiveram apenas a prematuridade com aqueles que apresentavam outros indicadores de risco associado ${ }^{24}$, não foi observada diferença estatisticamente significante entre os grupos (Tabela 1). Este dado demonstra que, na ausência de alteração auditiva, as células ciliadas externas do recém-nascido prematuro com indicador de risco associado ou não, apresentam semelhante funcionalidade.

$\mathrm{Na}$ análise comparativa da amplitude absoluta e nível de Response das EOE-t registradas nas orelhas direitas e esquerdas dos recém-nascidos observou-se diferença estatisticamente significante, com maior amplitude constatada na orelha direita, para ambos os grupos (Tabelas 2 e 3). Este dado corrobora os achados da literatura ${ }^{6-9}$, uma vez que apenas um estudo descreveu comportamento semelhante das emissões otoacústicas evocadas transientes independente da orelha ${ }^{11}$. A assimetria das emissões otoacústicas evocadas transientes obtidas entre as orelhas tem sido justificada pela atuação do sistema eferente auditivo sobre a amplitude das emissões otoacústicas ${ }^{10,26}$, assim como, a prevalência da presença das emissões otoacústicas espontâneas na orelha direita ${ }^{7}$. Ressalta-se, que não houve diferença da amplitude absoluta entre orelhas ${ }^{12,27}$, apenas, para a banda de frequência de $1 \mathrm{kHz}$, tanto para o grupo a termo quanto pré-termo, porém, não há nenhuma justificativa aparente para este achado.

Por outro lado, a amplitude absoluta por banda de frequência e do nível de Response das EOE-t mostrou-se semelhante nos recém-nascidos a termo e pré-termo, de acordo com as orelhas, não havendo diferença estatisticamente significante (Tabela 4). Apenas um estudo na literatura consultada apresentou a análise estatística comparativa entre a amplitude das emissões otoacústicas evocadas transientes obtidas em recém-nascidos prétermo e a termo, com diferença estatisticamente significante ${ }^{16}$, contrariando o resultado obtido no presente estudo. A prematuridade tem sido apresentada como um fator que influencia na amplitude das emissões otoacústicas, principalmente devido a variáveis não relacionadas à maturação coclear, como aspectos anatômicos e funcionais de orelhas externa e/ou média ${ }^{1-4}$. $\mathrm{Na}$ análise por frequência específica, pode-se observar que apenas a banda de frequência de $3 \mathrm{kHz}$ na orelha direita apresentou correlação estatisticamente significante em relação à prematuridade (Tabela 5).

A análise comparativa com e entre os estudos apresentados na literatura é complicada, uma vez que os mesmos adotam diferentes idades pós-concepcional na casuística. De maneira geral, a correlação mostrou-se significante quando o procedimento foi realizado em recém-nascidos com poucos dias de vida, período inicial com maior probabilidade de mudanças nas condições das orelhas externa e/ou média, principalmente no recém-nascido prétermo ${ }^{4}$ ou quando consideradas amplas faixas de idade ${ }^{6,16-21,23}$. No presente estudo, a idade pós-concepcional variou entre 24 horas de vida a 11 semanas, diferindo dos estudos descritos anteriormente.

Da mesma forma, quando a análise foi feita considerando o tempo de vida em horas foi evidenciada a existência de uma correlação significante desta variável com o registro das EOE-t em recémnascidos saudáveis, enfatizando que após 24 horas de vida o índice de não-passa na triagem auditiva reduziu significativamente ${ }^{25}$. Contudo, após 24 horas e considerando um período restrito, entre 24 e 54 horas, não se constatou esta correlação, o que levou os autores a reafirmar a recomendação da realização da triagem auditiva antes da alta hospitalar $^{22,23}$ (Tabelas 5 e 6).

\section{CONCLUSÃO}

As emissões otoacústicas evocadas transientes são passíveis de registro em recém-nascidos a termos e pré-termos, com semelhante amplitude absoluta e nível de Response, para a idade pósconcepcional analisada. 


\begin{abstract}
Purpose: to analyze the absolute amplitude and response level of transient evoked otoacoustic emissions in preterm and full-term newborns. Methods: 50 full-term and 50 preterm newborns took part in the study. They underwent newborn hearing screening, through research of transient evoked otoacoustic emissions, performed between 24 hours and 11 weeks of life. All newborns showed transient evoked otoacoustic emissions in the newborn hearing screening test. Results: no statistically significant difference of amplitude and response level was found between the full-term and preterm newborns. There was no significant correlation among factors such as prematurity, age of newborn at test and time of hearing stimulation with the results obtained. Conclusion: transient evoked otoacoustic emissions recorded in full-term and preterm newborns showed similar absolute amplitude and Response level.
\end{abstract}

KEYWORDS: Audiology; Neonatal Screening; Infant, Premature

\section{REFERÊNCIAS}

1. Tochetto T, Vieira EP. Legislação brasileira sobre triagem auditiva neonatal. Carapicuíba: Pró-Fono; 2006. 58p.

2. Marques TR, Mendes PC, Bochnia CFP, Jacob LCB, Roggia SM, Marques JM. Triagem auditiva neonatal: relação entre banho e índice de reteste. Rev Bras Otorrinolaringol. 2008; 74(3):375-81.

3. Tognola G, Parazzini M, Jager $P$, Brienesse $P$, Ravazzani P, Grandori F. Cochlear maturation and otoacoustic emissions in preterm infants: a time-frequency approach. Hear Res. 2005; 199(1-2):71-80.

4. Garcia CFD, Isaac ML, Oliveira JAA. Emissão otoacústica evocada transitória: instrumento para detecção precoce de alterações auditivas em recém-nascidos a termo e pré-termo. Rev Bras Otorrinolaringol. 2002; 68(3):344-52.

5. Driscoll C, Kei J, McPherson B. Transient evoked otoacoustic emissions in 6-year-old school children: a normative study. Scand Audiol. 2000; 29(2):103-10.

6. Bassetto MCA, Chiari BM, Azevedo MF. Emissões otoacústicas evocadas transientes (EOAET): amplitude da resposta em recém-nascidos a termo e pré-termo. Rev Bras Otorrinolaringol. 2003; 69(1):84-92.

7. Thornton ARD, Marotta N, Kennedy CR. The order of testing effect in otoacoustic emissions and its consequences for sex and ear differences in neonates. Hear Res. 2003; 184(1-2):123-30.

8. Saitoh $Y$, Sakoda $T$, Hazama M, Funakoshi $H$, Ikeda $H$, Shibano $A$, et al. Transient evoked otoacoustic emissions in newborn infants: effects of ear asymmetry, gender, and age. J Otolaryngol. 2006; 35(2):133-8.
9. Berninger E. Characteristics of normal newborn transient-evoked otoacoustic emissions: ear asymmetries and sex effects. Int J Audiol. 2007; 46(11):661-9.

10. Gkoritsa E, Korres S, Psarommatis I, Tsakanikos M, Apostolopoulos N, Ferekidis E. Maturation of the auditory system: 1 . Transient otoacoustic emissions as an index of inner ear maturation. Int $\mathrm{J}$ Audiol. 2007; 46(6):271-6.

11. Costa SMB, Costa Filho OA. Estudo das emissões otoacústicas evocadas em recémnascidos pré-termo. Pró-Fono. 1998; 10(1):21-5.

12. Kappor R, Panda NK. Transient evoked otoacoustic emissions. Indian J Pediatr. 2006; 73(4):283-6.

13. Ludovico AMAJ, Servilha BB. Perfil dos recémnascidos avaliados na triagem auditiva neonatal por meio de emissões otoacústicas evocadas por transitório. Rev Ciênc Méd. 2006; 15(2):14. Prieve BA, Hancur-Bucci CA, Preston JL. Changes in transient-evoked otoacoustic emissions in the first month of life. Ear Hear. 2009; 30(3):330-9.

15. Pinto VS, Lewis DR. Emissóes otoacústicas: produto de distorção em lactantes até dois meses de idade. Pró-Fono. 2007; 19(2):195-204.

16. Morlet T, Hamburger A, Kuint J, Ari-Even RD, Gartner M, Muchnik C, et al. Assessment of medial olivocochlear system function in pre-term and full-term newborns using a rapid test of transient otoacoustic emissions. Clin Otolaryngol Allied Sci. 2004; 29(2):183-90.

17. Brienesse $P$, Debyelann $P$. Maturation of otoacoustic emissions: longitudinal versus crosssectional study. Int J Pediatr Otorhinolaringol. 1997; 40(1):73-4. 
18. Kok MR, van Zanten GA, Brocaar MP. Growth of evoked otoacoustic emissions during the first days postpartum. A preliminary report. Audiology. 1992; 31(3):140-9.

19. Del Buono ZG, Mininni $F$, Delvecchio $M$, Pannacciulli C, Mininni S. Screening audiologico neonatale in prima e seconda giornata di vita. Minerva Pediatr. 2005; 57(4):167-72.

20. Barreira-Nielsen C, Futuro Neto HA, Gattaz G. Processo de implantação de programa de saúde auditiva em duas maternidades públicas. Rev Soc Bras Fonoaudiol. 2007; 12(2):99-105.

21. Trinidad Ruiz G, Pantoja Hernandez CG, Trinidad Ramos G, Serrano Berrocal MA, Pardo Romero G, Gonzalez Palomino A, et al. [Controlling retests in a universal hearing screening program]. Acta Otorrinolaringol Esp. 2005; 56(3):96-101.

22. Melo ADP, Alvarenga KF. Análise da ocorrência de falso positivo confirmado no teste-reteste da triagem auditiva neonatal. [monografia]. Bauru (SP): Instituto de Comunicação e Audição; 2005.
23. Melo ADP, Duarte JL, Alvarenga KF, AgostinhoPesse RS, Bevilacqua MC, Martinez MAN. Influência do tempo de vida na pesquisa das emissões otoacústicas evocadas transientes em recém-nascidos. Dist Comun. 2007; 19(3):357-64. 24. Joint Comittee on Infant Hearing $(\mathrm{JCIH})$. Year 2007 Position Statement: principles and guidelines for early hearing detection and intervention programs. Pediatrics. 2007; 120(4):898-921.

25. Sadri M, Thornton AR, Kennedy CR. Effects of maturation on parameters used for pass/fail criteria in neonatal hearing screening programmes using evoked otoacoustic emissions. Audiol Neurootol. 2007; 12(4):226-33.

26. Kuroda T. Clinical investigation on spontaneous otoacoustic emission (SOAE) in 447 ears. Auris Nasus Larynx. 2007; 34(1):29-38.

27. Mazlan R, Kei J, Hickson L, Stapleton C, Grant S, Lim S, et al. High frequency immittance findings: newborn versus six-week-old infants. Int $\mathrm{J}$ Audiol. 2007; 46(11):711-7.
RECEBIDO EM: 11/04/2008

ACEITO EM: 25/06/2009

Endereço para correspondência:

Kátia de Freitas Alvarenga

Departamento de Fonoaudiologia - Faculdade

de Odontologia de Bauru - Universidade de São

Paulo

Al. Octávio Pinheiro Brisolla, 9-75

Bauru - SP

CEP: 17012- 901

E-mail: katialv@fob.usp.br 